\title{
Battered Women's Coping Strategies and Psychological Distress: Differences by Immigration Status
}

\author{
Mieko Yoshihama ${ }^{1}$ \\ University of Michigan School of Social Work
}

People are rarely passive, and battered women are no exception. This study investigated the types of coping strategies women of Japanese descent (both Japan-born and U.S.-born) chose and their perceived effectiveness in dealing with their partners' violence. Japan-born respondents were significantly less likely to use "active" strategies and perceived them to be less effective than did U.S.-born respondents. For the Japan-born, the more effective they perceived "active" strategies, the higher their psychological distress, whereas the more effective they perceived "passive" strategies, the lower their psychological distress. In contrast, for the U.S.-born, the higher the perceived effectiveness of "active" strategies, the lower their psychological distress, and the perceived effectiveness of "passive" strategies had little effect on their psychological distress. The complex relationship between individuals' country of birth, the choice and perceived effectiveness of coping strategies, and psychological distress calls for increased attention to the role of culture in studies of coping and domestic violence.

KEY WORDS: coping; domestic violence; psychological distress; immigration status.

\section{INTRODUCTION}

People are rarely passive, and battered women are no exception. Women cope with domestic violence in a variety of ways, such as seeking information, redefining the problem/situation, seeking help from family, friends, and legal

\footnotetext{
${ }^{1}$ To whom correspondence should be addressed at University of Michigan School of Social Work, 1080 S. University, Ann Arbor, Michigan 48109-1106; e-mail: miekoy@umich.edu.
} 
or social service organizations, and leaving the relationship temporarily or permanently (Bowker \& Maurer, 1986; Horton \& Johnson, 1993; Kelly, 1988; Mitchell \& Hodson, 1983; Nurius, Furrey, \& Berliner, 1992; Sullivan, 1991). Coping is a shifting, not static, process in that individuals employ varying strategies according to the nature of the stressors, appraisal of the available coping resources, and other situational factors (Folkman, 1984; Folkman \& Lazarus, 1980; Pearlin \& Schooler, 1978). Using a community-based random sample, this study investigated the selection and perceived effectiveness of coping strategies among women of Japanese descent who had experienced partners' violence. In particular, this paper examined whether the individuals' country of birth was associated with the selection and perceived effectiveness of coping and what relationship, if any, existed between the selection and perceived effectiveness of coping and the level of psychological distress.

In studies of battered women, coping strategies are typically dichotomized as "active versus passive": observable, behavioral efforts are categorized as "active" strategies, and unobservable, cognitive or emotional efforts, as "passive" strategies (Finn, 1985). Others have classified battered women's coping as "engagement" versus "disengagement" (Kemp, Green, Hovanitz, \& Rawlings, 1995). One problem associated with studies of battered women's coping is a reliance on respondents recruited through shelters and other social service programs. These studies have generally found the use of "active" or "engagement" strategies to be associated with a lower level of psychological distress, and the use of "passive" or "disengagement" strategies, with a higher level of psychological distress (e.g., Kemp et al., 1995; Mitchell \& Hodson, 1983). This finding is consistent with that of studies of coping among general populations (Holahan \& Moos, 1990, 1991). Caution is required, however, in generalizing the relative effectiveness of "active" strategies among convenience samples of battered women, because such studies contain an inherent sampling bias for women who have contacted outside agencies, who are by definition "active copers."

The positive evaluation of "active" coping strategies overlooks some of the important negative consequences such strategies may have for battered women. For example, women who attempt to leave their partners are at higher risk for being murdered (Wilson \& Daly, 1993). Thus, attempts to leave, a strategy conventionally considered "active," may be contraindicated in certain situations. The use of "passive" strategies may reflect a battered woman's deliberate choice based on her accurate assessment of the situation and available options, as opposed to a deficiency in "active coping" skills. Browne (1993) points out:

Behaviors that outside observers may interpret as helplessness-such as staying with the abuser or refraining from initiating legal actions against him-may simply be accurate evaluations of the assailant's potential for violent responses and others' inability to intervene in time to guarantee safety. (p. 1080) 
Therefore, it is important to understand the factors that moderate a battered woman's choice and effectiveness of coping strategies and assess their effectiveness in the specific situational context.

\section{Goodness-of-Fit Theories of Coping: Adding Culture to the Equation}

In general, research on coping has found that the perceived controllability of the stressful situation influences individuals' choice of coping strategies (Folkman, 1984; Valentiner, Holahan, \& Moos, 1994). People are likely to use emotion-focused strategies when they perceive the situation as uncontrollable, whereas if the situation is perceived as controllable, people tend to resort to problem-focused strategies (Folkman, Schaefer, \& Lazarus, 1979; Forsythe \& Compas, 1987; Moos \& Schaefer, 1993). This model, referred to as the goodness-of-fit hypothesis, may help explain battered women's use of emotion-focused or "passive" strategies in the face of an uncontrollable situation - their partners' violence. However, research findings are, thus far, inconclusive with respect to the fit between coping strategies and the controllability of the situation among battered women. One recent study, which investigated women's appraisal of controllability of partners' violence and the choice of coping strategies among a convenience sample of college students, found no significant relationship between the two (Pape \& Arias, 1995). The researchers speculated that a sense of shock and confusion at the time of victimization might have impeded women's ability to rely on appraisal of the situation in selecting coping strategies that matched the nature of the stressor. On the other hand, Hamby and Gray-Little (1997) found that the more severe the violence, the more "active" and the less "passive" strategies were used among a sample of mostly white middle-class women. It is plausible that factors other than the perceived controllability of the stressful situation influenced women's choice of coping strategies.

An important variable that has been overlooked in studies of coping is culture. Cultural values and norms may influence battered women's choices, prescribing the range of coping strategies available or acceptable to them. In some cultures, the use of certain "active" strategies may be discouraged. For example, leaving the relationship may not be viable for some women because of religious or cultural reasons. Such acts may incur serious familial or societal sanctions, such as being ostracized by members of their families or community. For some, confronting their partners, asserting their rights, or seeking outside assistance may mean going against their cultural norms and values. A pressure to acquiesce, to place collective welfare and family harmony over one's individual needs and rights, and to save the family's face may be stronger in some cultures than in others. Thus, some women may appear "passive" in their victimization because of such cultural prescriptions. 
A recent study of battered women of Japanese descent in the United States found cultural factors (e.g., the value placed on endurance, "face saving" especially in relation to the family's honor, conflict avoidance, and preserving family harmony at all costs) hindered their recognition of partners' behavior as abusive and impeded their help-seeking efforts (Yoshihama, 2000). These values and norms were passed down and reinforced, verbally or otherwise, within their families and were strong even for those U.S.-born Japanese American women whose families had lived in the United States for several generations. Other qualitative studies have similarly documented various ways in which cultural values and norms, religious teachings, and racism influence battered women's coping, especially women of color (Allen, 1986; Ginorio \& Reno, 1986; Ho, 1990; Lai, 1986; Richie \& Kanuha, 1993; Torres, 1987).

These findings point to the potentially significant effect of cultural factors on the selection and perceived effectiveness of coping strategies a woman has employed. In addition, previous studies have found a significant relationship between the individual's selection of coping strategies and mental health in the general population (Holahan \& Moos, 1990, 1991) and in battered women (Kemp et al., 1995; Mitchell \& Hodson, 1983). It is possible that culture moderates such a relationship; nonetheless, little research has been conducted in this area.

Even when researchers include culture in investigations, they tend to treat culture as a homogeneous variable. Consequently, most cross-cultural investigations have focused on between-group differences and have tended to aggregate individuals into broad racial groups regardless of their ethnicity or country of origin. For example, individuals of various Hispanic backgrounds are often lumped together as Hispanics or Latinos/Latinas. Similar aggregation is also common for studies of women of Asian and Pacific Islander backgrounds who consist of different ethnic and cultural groups (e.g., Chinese, Filipina, Japanese, and Vietnamese). In addition, many studies aggregate individuals regardless of their immigration status, such as country of birth and generational position. Such aggregation runs the risk of overlooking significant within-group variations (Uehara, Takeuchi, \& Smukler, 1994). Examination by immigration status or country of birth often uncovers significant within-group differences in women's experiences of domestic violence (Kantor, Jasinski, \& Aldarondo, 1994; Sorenson \& Telles, 1991). For example, the rate of domestic violence victimization was significantly higher among U.S.-born Mexican Americans women than among Mexicans residing in the United States (Kantor et al., 1994).

One unique feature of the present study is the use of respondents who share a common cultural background but were born in different countries, allowing for an exploration of within-group differences. It is hoped that 
the attention to within-group variations in the choice and perceived effectiveness of coping strategies will contribute to the development of more integrated theories of stress-coping-distress relationships in general, and those for battered women of color in particular. Specifically, this study examined the following hypotheses:

1. Controlling for the characteristics of partners' violence, the individuals' country of birth has a significant effect on the selection and the perceived effectiveness of coping strategies. For example, it was hypothesized that U.S.-born women were more likely to confront abusive partners and to seek outside help than Japan-born women.

2. The level of psychological distress is inversely associated with the perceived effectiveness, but not the type, of coping strategies, and the individuals' countries of birth moderates this relationship.

\section{METHODS}

\section{Respondents}

Respondents in this study were 129 women who reported having experienced partners' violence in a face-to-face interview study that investigated the prevalence of domestic violence. The overall interview study employed a community-based random sample of 211 women of Japanese descent selected from a list of households containing persons with a Japanese surname in Los Angeles County. Following an introductory letter sent to the households, a screening telephone call was made to the household to identify any woman who met the following criteria: Japanese descent, born in the United States or Japan, ages between 18 and 49, and having had an intimate heterosexual relationship. Only one woman per household was selected using a random procedure. Structured face-to-face interviews were conducted by trained interviewers, who were matched to the respondent's preferred language (English or Japanese). The questionnaire was developed using a decentering method (Marin \& Marin, 1991) to ensure that the Japanese and English versions were comparable linguistically as well as conceptually. This method involved developing a questionnaire in two languages simultaneously, followed by a series of translation between the two versions as revisions were made to either version. Two versions of the questionnaire were repeatedly reviewed for their conceptual and linguistic equivalency by a group of practitioners, researchers, and graduate students. Subsequently, both versions of the questionnaire were pilot-tested.

Of the 407 households determined to have at least one eligible woman, 211 women completed the interview-a response rate of $52 \%$; this rate is 
comparable to other studies of women's victimization that employed similar in-depth, face-to-face interviews (Russell \& Howell, 1983; Wyatt, 1985) or telephone interviews (Smith, 1987). The 211 participants of the overall study did not differ from those who declined to participate and were comparable to female residents of Japanese descent aged 18-49 in Los Angeles County based on the Census data. Written consent was obtained at the beginning of the interview. In addition to approval from the Institutional Review Board, a Certificate of Confidentiality was obtained from the National Institute of Mental Health, Department of Health and Human Services. The interview was audiotaped if the respondent consented (67\% of the cases). On average, the interviews lasted $90 \mathrm{~min}$. At the end of the interview, the respondent received $\$ 20$ and a list of available assistance programs, including services provided in Japanese. All respondents were asked whether they wished to speak to a counselor, and a referral for counseling was made if needed.

Because the history of domestic violence victimization was not a sampling criterion, the sample included both women who had been abused and those who had not. It was only after the questions regarding victimization history were asked during the interview that women who had been abused were identified $(n=129,61.1 \%)$. All of the 129 women who had been abused completed an additional section of the interview, which pertained to the characteristics of the perpetrator, the respondent's coping, and other aspects of her victimization experience. Those 129 women did not differ from those who did not report abuse with respect to educational level, employment status, income, or country of birth.

\section{Measurements}

\section{Characteristics of Domestic Violence Victimization}

Using behavior-specific questions, the respondent was asked whether she had ever experienced each of 62 types of violence at the hands of her current or former partners. These items were drawn from various studies in the United States and Japan (Marshall, 1992; Straus \& Gelles, 1986; Tolman, 1989; Yoshihama \& Sorenson, 1994) and covered a wide range of violent acts of a physical, emotional, or sexual nature, ranging from pushing to use of lethal weapons, from verbal debasement to restriction of activities and threatening behaviors, and from unwanted touching to forced intercourse (see Yoshihama, 1999, for specific types of violence examined). For each type of violence ever experienced, the respondent was asked to indicate 
its perceived abusiveness on a 4-point Likert-type scale ranging from 1, not at all abusive to 4, very abusive. This measurement of "abusiveness," based on the respondent's own assessment, diverges from that of the "severity" of violence in previous studies where the severity of each specific violent behavior is predetermined (Hudson \& McIntosh, 1981; Marshall, 1992; Straus, 1990).

An indicator of the abusiveness ratings of domestic violence over the course of the respondent's lifetime was measured by summing perceived abusiveness ratings. Because some women had been abused by more than one partner over the course of their lives, the summed abusiveness rating scores may include acts perpetrated by more than one partner. If there were multiple perpetrators, the characteristics of the one who perpetrated the most abusive incident (the indexed partner, hereinafter) was obtained; the most abusive incident was determined by the respondent as part of the interview. In addition to the racial or ethnic background of the perpetrator (or both), the respondent who had child(ren) with the indexed partner was asked whether he abused the children. The respondent was also asked whether she had ever sustained injuries due to a partner's violence, and if so, how many times during her lifetime and during the past year.

\section{Types and Perceived Effectiveness of Coping Strategies}

Both the choice and perceived effectiveness of coping strategies were assessed. The respondent was asked whether she used each of 13 specific strategies in dealing with the violence perpetrated by the (indexed) partner. Specific strategies examined included seeking assistance from the family, friends, or counselors; confronting the partner; leaving the relationship; redefining the meaning of the situation; and the use of alcohol and drugs (Cronbach's $\alpha=.58$ for U.S.-born and .65 for Japan-born respondents). These items were developed based on previous studies (e.g., Billings \& Moos, 1981; Finn, 1985; Mitchell \& Hodson, 1983; Nurius et al., 1992) and the author's practice experience with battered women of Japanese descent. For each strategy the respondent used, she was asked to rate its effectiveness on a 4-point Likert-type scale: $1=$ not at all helpful, $2=$ not so helpful, $3=$ somewhat helpful, and $4=$ very helpful.

\section{Country of Birth}

Country of birth was a dichotomous variable, with those born in the United States coded 1, and Japan-born coded 0. 


\section{Psychological Distress}

An indicator of current level of overall psychological distress was measured by the Global Severity Index of the Symptoms Check List-90R (SCL-90R GSI; Derogatis, 1994). This measure was chosen based on its validity and reliability with the Japanese American population (Takeuchi, Kuo, Kim, \& Leaf, 1990) and also due in part to the availability of a version translated into Japanese. The SCL-90R GSI was calculated by dividing the sum score of all 90 symptoms by the total number of responses (possible scores range from 0 to 4): This score combines information concerning the range of psychological distress (e.g., the number of symptoms experienced) with that of the intensity of distress (Derogatis, 1994). A higher score indicated a higher level of distress during the previous week. The internal consistencies for the nine specific dimensions for U.S.-born respondents (Cronbach's $\alpha=.80$ to .91 ) and those for Japan-born respondents (Cronbach's $\alpha=.76$ to .94 ) were comparable to those reported by the scale's authors (Cronbach's $\alpha=.77$ to .90; Derogatis, Richels, \& Rock, 1976). Because the distribution of psychological distress was skewed, log transformation was used.

\section{Control Variables}

The respondent's age was included as a control variable in examining the relationship between country of birth and the level of psychological distress. The inclusion of this control variable was based on a previous study using the same sample of respondents, as well as other epidemiologic studies that found a strong relationship between psychological distress and age (Kessler et al., 1994; Robins, Locke, \& Regier, 1991; Yoshihama, 2001).

\section{Analysis}

Descriptive statistics were used to examine the characteristics of the respondents, domestic violence victimization, and type and perceived effectiveness of the coping strategies used. Using Chi-square statistics, the proportions of U.S.-born and Japan-born women who used specific types of coping strategies were compared, and a $t$ test was used to examine differences in their perceived effectiveness between the two groups of women. Prior to multivariate analyses, data reduction was conducted using a principal component factor analysis (varimax rotation) on the 13 types of coping strategies 
used by respondents (binomial responses as to whether the respondent used a specific type of coping strategies).

Multiple regression analyses were used to examine whether country of birth was associated with the selection and perceived effectiveness of coping strategies, controlling for the characteristics of domestic violence. An additional multiple regression analysis investigated the relationship between the type and perceived effectiveness of coping strategies and psychological distress. A moderation effect of country of birth was examined by including interaction terms in the regression model. All multiple regression analyses used the ordinary least square method and forced entry method.

\section{RESULTS}

\section{Respondents' Characteristics and Experiences of Partners' Violence by Country of Birth}

The average age of the respondents was 37 years (Table I). The majority had a college education and were working full-time. Their personal incomes varied considerably: $43 \%$ had annual personal income of less than $\$ 15,000$, whereas approximately one third earned $\$ 40,000$ or more annually. The majority were married or in a committed relationship at the time of the interview. Sociodemographically, U.S.-born and Japan-born respondents were similar, except that the former was significantly younger than the latter, $t=3.43, p<.05$.

The summed abusiveness ratings varied considerably, with one fourth scoring 30 or higher. Approximately one in eight had been injured owing to partners' violence, and $47 \%$ of those had been injured multiple times. One in ten (or $23 \%$ of those who had had children with an abusive partner) reported that their partners also abused their children. No differences by country of birth were found in these characteristics of domestic violence victimization. However, a significantly higher proportion of U.S.-born respondents $(50 \%)$ than Japan-born respondents (17\%) were in an interethnic or interracial relationship, $\chi^{2}=10.20, p<.05$. No difference by country of birth was found in the level of current psychological distress.

\section{Coping Strategies Employed and Their Perceived Effectiveness}

As Table II indicates, women of Japanese descent in the present study used a wide range of coping strategies in dealing with partners' violence, 


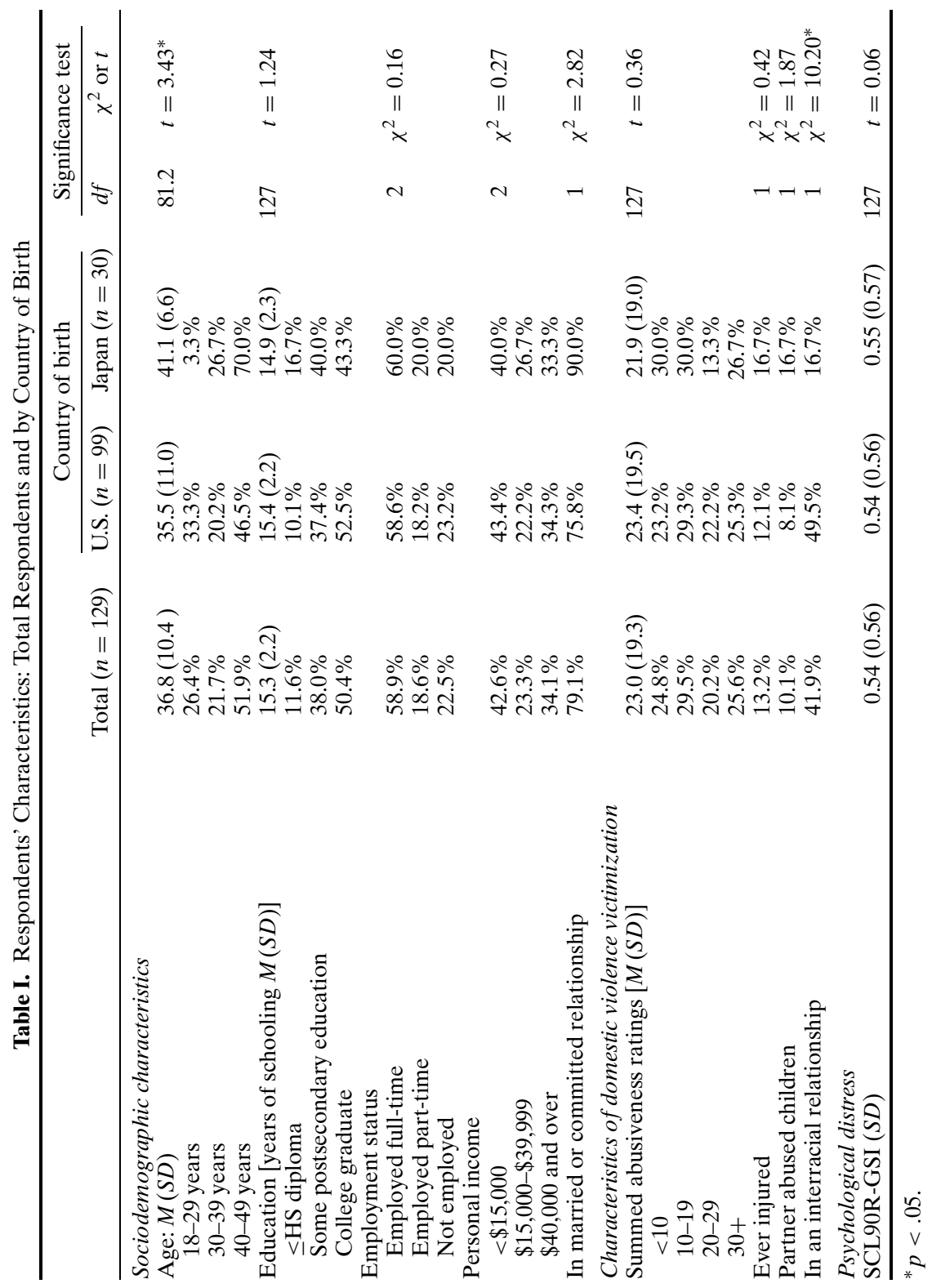




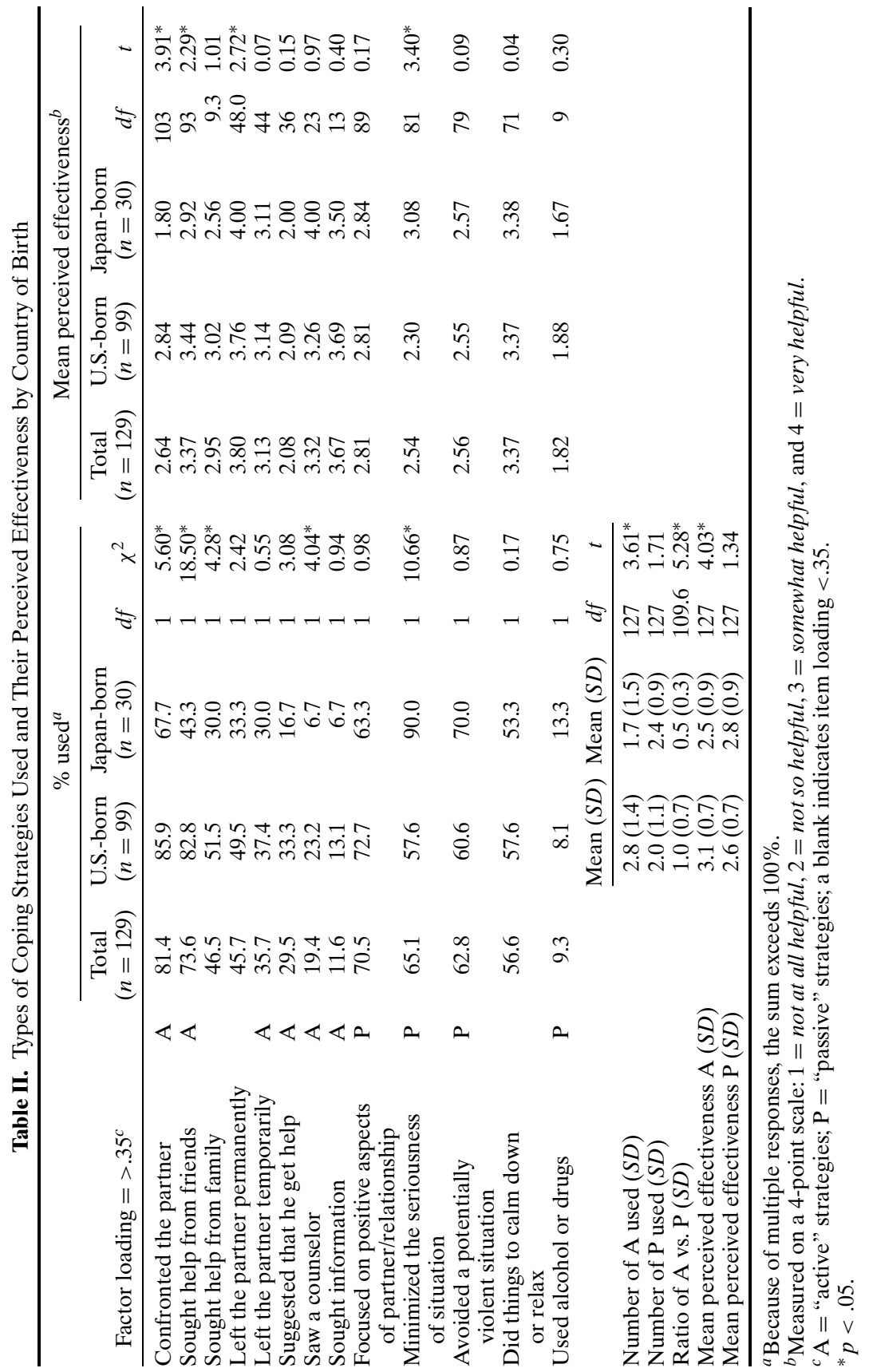


and often used multiple strategies. Confronting the partner (81\%) and seeking help from friends $(74 \%)$ were reported by the largest proportions of the respondents. The perceived effectiveness of specific strategies varied, and those strategies used by a large proportion of the respondents were not necessarily rated highly. For example, although the majority confronted their partner, many of them considered it "not so helpful" $(M=2.6)$. Similarly, although the majority redefined the situation by focusing on the positive aspects of the partner/relationship or minimizing the seriousness of the situation, many found these strategies not so helpful $(M=2.8$ and 2.5 , respectively). Doing things to calm down or relax, in contrast, was rated more helpful on average $(M=3.4)$. Seeking information and seeing a counselor, albeit used by a small number, were perceived as helpful on average.

Some significant differences were found in the choice and perceived effectiveness of coping strategies between U.S.-born and Japan-born respondents. U.S.-born respondents were significantly more likely to seek help from friends and to confront the partner, but significantly less likely to minimize the seriousness of the situation than were their Japan-born counterparts. A similar pattern was found in the perceived effectiveness of those strategies by country of birth. U.S.-born respondents perceived seeking help from friends as significantly more helpful, but minimizing the seriousness of the situation as significantly less helpful, than did Japanborn respondents. Although confronting the partner was rated significantly higher by U.S.-born respondents than by Japan-born respondents, its effectiveness was less than somewhat helpful for both groups of women ( $M=2.8$ for the U.S.-born and $M=1.8$ for the Japan-born; $t=3.91$, $p<.05)$.

A principal component factor analysis of the 13 types of coping strategies found two factors, which explained $31 \%$ of total variance. Items loading .35 or higher on the first factor (Eigenvalue $=2.35$ ) were confronting the partner, seeking help from friends $(\alpha=.62)$, leaving temporarily, suggesting that the partner seek help, seeing a counselor, and seeking information. This factor generally corresponds to strategies conventionally categorized as "active." The second factor (Eigenvalue $=1.69$ ) consisted of four items loading .35 or higher: focusing on the positive aspects of the partner or the relationship ( $\alpha=.42$ ), minimizing the seriousness of the situation, avoiding potentially violent situations, and using alcohol or drugs. This factor corresponds to "passive" strategies.

U.S.-born respondents used an average of 2.8 "active" strategies $(S D=1.4)$, a significantly larger number than did Japan-born respondents ( $M=1.7, S D=1.5$; Table II, lower portion $)$. No significant differences were 
found between U.S.-born and Japan-born women in the number of "passive" strategies. The ratio of "active" versus "passive" strategies used was measured by dividing ([the number of "active" strategies used +1$] / 6$ ) by ([the number of "passive" strategies used +1$] / 4$ ). A constant value of 1 was added to the numerator in order to ensure the inclusion of a small number of respondents who used none of the "active" or "passive" strategies. Two composite scores were also created: (1) perceived effectiveness of "active" strategies by averaging the effectiveness ratings of six "active" strategies, and (2) perceived effectiveness of "passive" strategies by averaging the effectiveness ratings of four "passive" strategies. The "active" versus "passive" ratio was 1.0 for U.S.-born and .5 for Japan-born respondents $(t=5.28, p<.05)$; U.S.-born respondents used both "active" and "passive" coping strategies equally, whereas Japan-born respondents were nearly two times more likely to use "passive" than "active" strategies. U.S.-born respondents considered the "active" strategies they employed more effective than did Japan-born women $(M=3.1$ and 2.5, respectively. No significant bivariate difference was found in the perceived effectiveness of "passive" strategies between U.S.-born and Japan-born women.

Multiple regression analysis was performed to examine whether the selection and the perceived effectiveness of coping strategies were associated with country of birth, controlling for the characteristics of domestic violence victimization. Because three variables of victimization characteristics (i.e., summed abusiveness ratings, experience of injuries, and the partner's abuse toward children) were significantly correlated, only the summed abusiveness ratings was entered into the regression model. Age and whether the respondent was in an interracial relationship (interracial relationship, hereinafter) were also included in the model because U.S.-born respondents were significantly younger and more likely to have been in an interracial relationship than Japan-born respondents. Bivariate relationships of these variables are presented in Table III.

As shown in Table IV, country of birth was significantly associated with the ratio of "active" versus "passive" strategies. U.S.-born respondents were significantly more likely to use "active" strategies and perceive them as more effective than Japan-born respondents. After controlling for age and the abusiveness ratings of domestic violence, the respondents' country of birth was not associated with the perceived effectiveness of "passive" strategies. Also notable was that the abusiveness ratings of domestic violence were not associated with the selection of coping strategies; however, they were negatively associated with the perceived effectiveness of both types of coping strategies. 


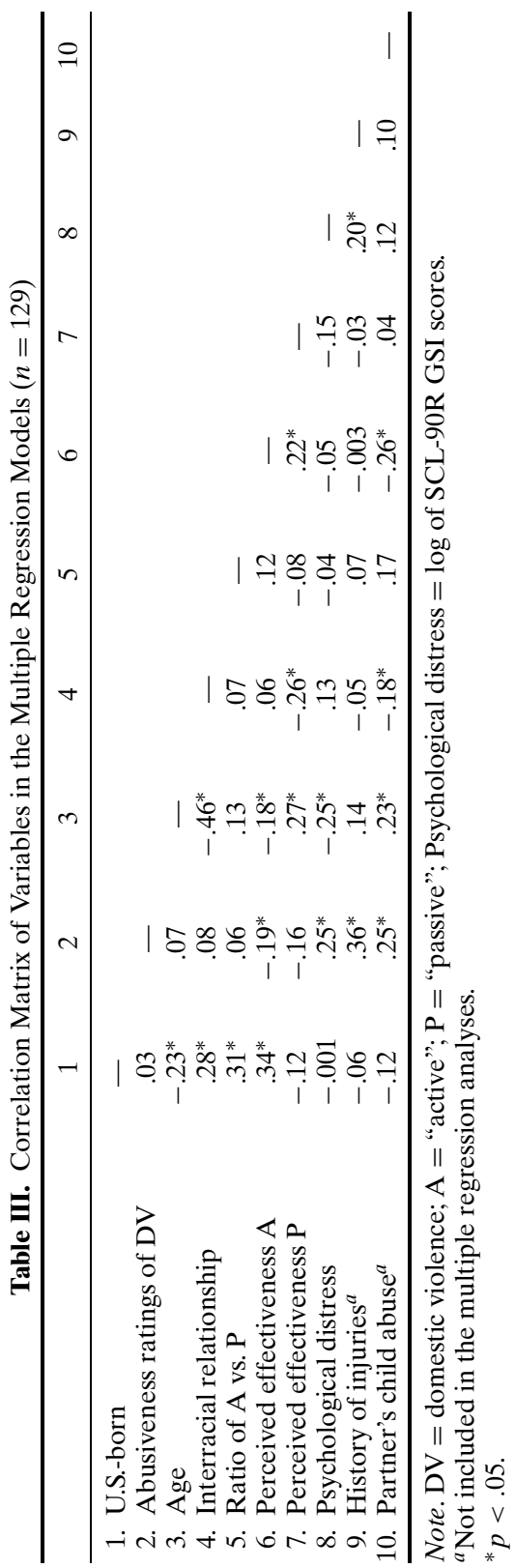


Table IV. Multiple Regression Analysis Predicting the Ratio of "Active" Versus "Passive" Strategies and Their Perceived Effectiveness $(n=129)$

\begin{tabular}{|c|c|c|c|c|c|c|c|c|c|}
\hline & \multicolumn{3}{|c|}{ Ratio of A vs. P } & \multicolumn{3}{|c|}{$\begin{array}{c}\text { Perceived } \\
\text { effectiveness of A }\end{array}$} & \multicolumn{3}{|c|}{$\begin{array}{c}\text { Perceived } \\
\text { effectiveness of } \mathrm{P}\end{array}$} \\
\hline & $\bar{\beta}$ & $S E$ & $B$ & $\beta$ & $S E$ & $B$ & $\beta$ & $S E$ & $B$ \\
\hline $\begin{array}{l}\text { Summed abusiveness } \\
\text { ratings of DV }\end{array}$ & .001 & .003 & .03 & -.008 & .003 & $-.19^{*}$ & -.007 & .003 & $-.17^{\dagger}$ \\
\hline Age & .014 & .006 & $.24^{*}$ & -.009 & .007 & -.12 & .02 & .007 & $.21^{*}$ \\
\hline $\begin{array}{l}\text { Interracial } \\
\quad \text { relationship }\end{array}$ & .10 & .12 & .08 & -.13 & .15 & -.08 & -.22 & .15 & -.14 \\
\hline U.S.-born & .49 & .13 & $.34^{*}$ & .64 & .16 & $.34^{*}$ & -.05 & .16 & -.03 \\
\hline $\begin{array}{l}\text { Model } F(4,124) \\
R^{2} \\
\text { Adjusted } R^{2}\end{array}$ & & $\begin{array}{c}5.04^{*} \\
.14 \\
.11\end{array}$ & & & $\begin{array}{l}6.17^{*} \\
.17 \\
.14\end{array}$ & & & $\begin{array}{l}4.35^{*} \\
.12 \\
.10\end{array}$ & \\
\hline
\end{tabular}

Note. $\mathrm{A}=$ "active" strategies; $\mathrm{P}=$ "passive" strategies; $\mathrm{DV}=$ domestic violence.

$* p<.05$.

${ }^{\dagger} p<.10$.

\section{The Relationship Between Coping and Psychological Distress by Country of Birth}

Using the block entry method, multiple regression analysis was performed to investigate whether level of psychological distress was related to the selection and perceived effectiveness of coping strategies, and whether this relationship was moderated by country of birth (see Table V). Marginal relationships among those variables and control variables included in the model are presented in Table III. Age was entered first (Column I), followed by the summed abusiveness ratings of domestic violence (Column II). The summed abusiveness ratings of domestic violence was significantly associated with psychological distress. The ratio of "active" versus "passive" strategies and the perceived effectiveness of "active" and "passive" strategies were entered next (Column III), none of which were significantly related to the level of current psychological distress. In the next block, country of birth was not associated with the level of psychological distress (Column IV).

When interaction terms between country of birth and the perceived effectiveness of "active" and "passive" strategies were entered (Column V), the model explained an additional $7 \%$ of the variance. There was a significant main effect of the effectiveness of "passive" strategies $(B=-.37)$, as well as significant interaction effects between country of birth and the perceived effectiveness of both "active" and "passive" strategies $(B=-.37$ and $B=.41$, respectively). The relationships between psychological distress and perceived effectiveness of "active" and "passive" strategies by country of birth are presented in Fig. 1. For the U.S.-born, the higher the perceived 


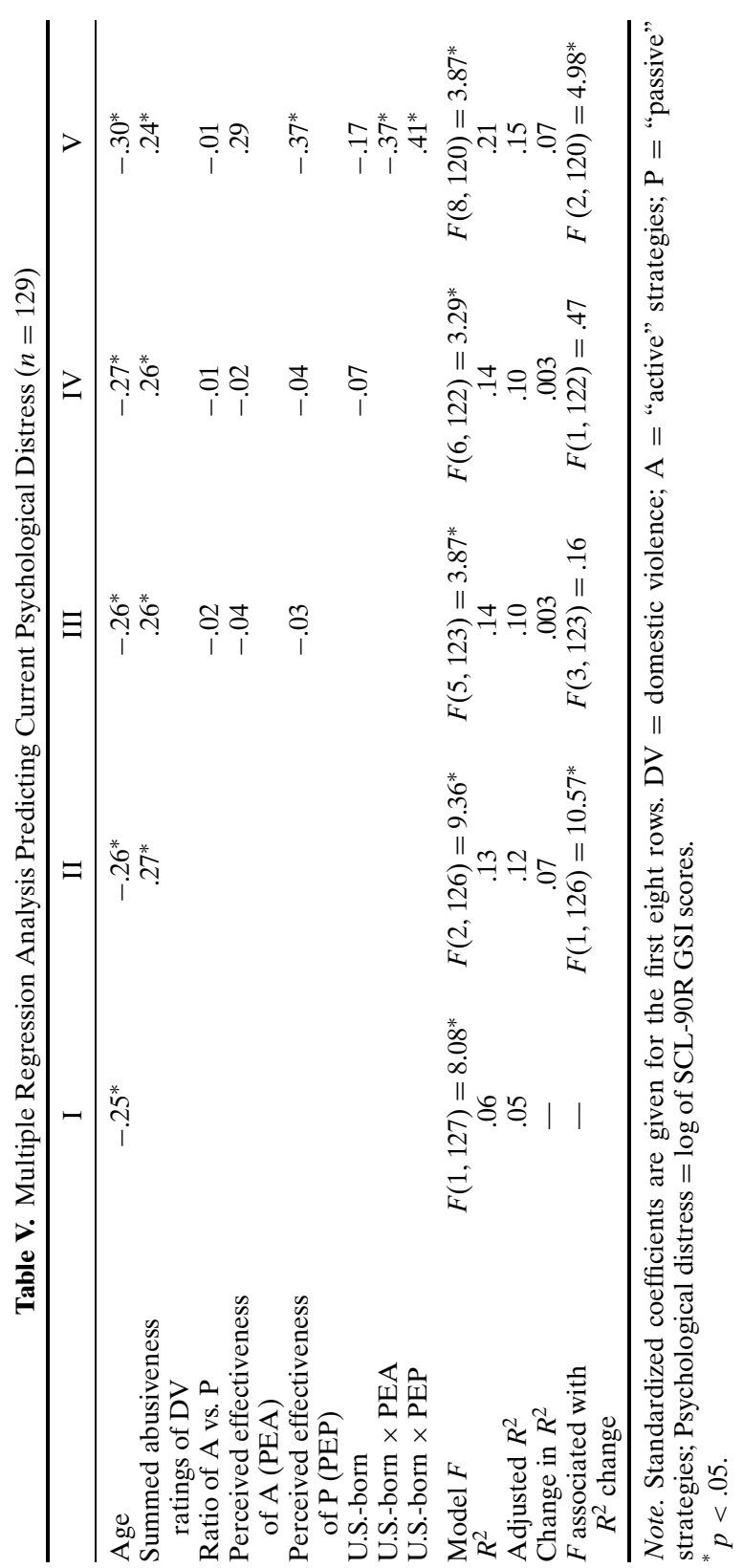



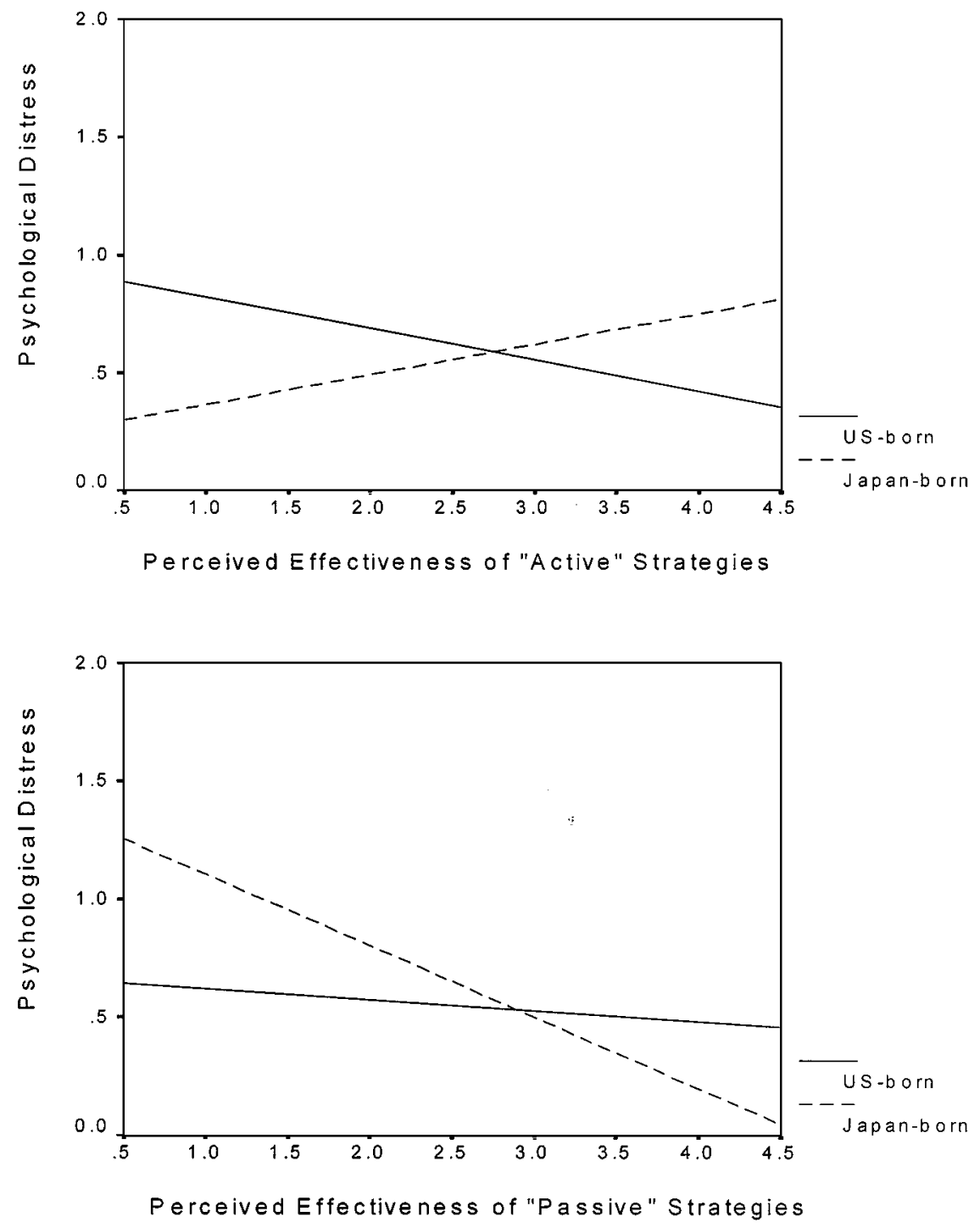

Fig. 1. Psychological distress and perceived effectiveness of "active" and "passive" coping strategies by country of birth. 
effectiveness of "active" strategies, the lower their psychological distress. Interestingly however, for the Japan-born, the higher the effectiveness of "active" strategies, the higher their psychological distress. Although the perceived effectiveness of "passive" strategies did not affect their psychological distress for the U.S.-born, for the Japan-born, the higher the perceived effectiveness of "passive" strategies, the lower their psychological distress.

\section{DISCUSSION}

This study began by questioning the role of culture in the selection and perceived effectiveness of coping strategies among battered women. The use of "active" or "passive" strategies was found to be related to the respondents' country of birth. Compared to Japan-born counterparts, U.S.-born respondents were more likely to use "active" strategies and tended to perceive them as more effective. The strong association between country of birth and the ratio of "active" versus "passive" strategies was present, after controlling for age and the abusiveness of domestic violence.

This study's findings present a rather complex relationship among individuals' country of birth, behavior choice (e.g., the ratio of "active" vs. "passive" strategies), its meaning (e.g., perceived effectiveness), and psychological distress. The U.S.-born and Japan-born respondents differed significantly with respect to choice of "active" versus "passive" strategies and perceived effectiveness of "active" strategies. Lesser use of strategies such as confrontation and help-seeking from friends and the significantly lower effectiveness rating of those strategies among Japan-born respondents may reflect a stronger degree of cultural proscription against these "active" acts for the Japan-born. The result of multiple regression analysis provides additional support for the existence of stronger cultural proscriptions against "active" strategies for Japan-born respondents. For Japan-born respondents, the more effective they perceived "active" strategies, the worse off they were psychologically. Interestingly, the opposite was true for the U.S.-born: the more effective they perceived "active" strategies, the lower their psychological distress. With respect to the perceived effectiveness of "passive" strategies, it had little effect on psychological distress among the U.S.-born, whereas for the Japan-born, the more effective they perceived "passive" strategies, the better off they were psychologically. These opposite or different effects by country of birth may explain why the perceived effectiveness of "active" and "passive" strategies were not significant when interaction terms were not entered in the model.

These findings challenge the simplicity of an active-passive dichotomous classification of battered women's coping strategies. It appears that 
a match between cultural proscriptions and coping strategies, rather than the choice of strategies itself, serves as a protective factor for psychological distress among battered women who were born in Japan. The rather paradoxical relationship between the perceived effectiveness of "active" strategies and psychological well-being among Japan-born women may involve both direct and indirect pathways (A. Browne, personal communication, July 27, 1999). The use of "active" coping strategies, such as confronting and divorcing the abusive partner, in and of itself may be detrimental to the psychological well-being of Japan-born women, because such strategies are culturally incongruent. Indirectly, the use of such "active" coping strategies may be frowned upon by family members and friends. This may result in reduced social support, which in turn may be associated with decreased psychological well-being. Additional qualitative studies may shed light on specific pathways that may underlie the observed relationship between the selection and perceived effectiveness of coping strategies, psychological distress, and the country of birth.

\section{Implications for Practice, Policy, and Research}

Frequently used strategies were not necessarily rated as being effective. "Active" strategies targeting the partner, such as confronting him and suggesting that he seeks help, were rated as not very effective, whereas others, such as seeing a counselor and seeking information, were rated helpful by the majority. In assessing a battered woman's coping strategies, attention should be paid not only to the type of strategies used, but also to their subjective meaning in the specific situational context. As the findings of this study suggest, the perceived effectiveness of "passive" strategies may serve as a protective factor for psychological distress for Japan-born women. The use of "passive" strategies should not be viewed as a deficiency. Encouraging battered women, especially Japan-born women, to use "active" coping strategies may not necessarily alleviate their distress. The perceived effectiveness of coping strategies needs to be interpreted in the sociocultural context, and culturally congruent coping styles need to be viewed as a source of strength.

The results of this study suggest the importance of the congruence between choice of strategies and cultural proscriptions, in that this cultural congruence influences the perceived effectiveness of the coping strategies used by battered women, which in turn affects their level of psychological distress. To alleviate or minimize emotional suffering in battered women, interventions must be designed to promote their use of culturally congruent coping strategies when possible. The qualifier of "when possible" reflects the reality that the potentially lethal nature of domestic violence may at times require a woman to employ drastic actions, such as leaving the relationship 
or calling the police, no matter how culturally incongruent these actions may be. Professional consultation is important for practitioners who are not familiar with the cultural background of the battered woman with whom they are working. Developing support groups for battered women who share a common cultural background may facilitate collective validation of their culturally influenced coping styles as a strength. Although interventions aimed at promoting culturally congruent coping may help reduce the level of psychological distress among battered women, given the significant effect of the abusiveness ratings of domestic violence victimization on women's psychological distress, alleviation of psychological distress ultimately requires preventing victimization.

In the United States, interventions for domestic violence have been primarily focused on the response by the criminal justice system and on women taking action, such as calling a hotline and seeking shelter (Mills, 1999; Schechter, 1996). In certain states, health care professionals are mandated to report cases of domestic violence to law enforcement (Hyman, Schillinger, \& Lo, 1995). In response to domestic violence cases, pro-arrest and pro-prosecution policies, and mandatory arrest policies in some areas, have been implemented in an increasing number of locations (Zorza \& Woods, 1994). Battered women are encouraged to call domestic violence hotlines and the police and are asked, under subpoena at times, to testify against the perpetrators-an approach contradictory to some women's cultural proscriptions. The findings of this study suggest that such an approach may result in higher psychological distress for some women-immigrant women from Japan in particular. Although mental health services may help alleviate psychological distress among women who have taken "active" coping strategies (e.g., testifying as a witness against her partner), current reliance on criminal justice interventions in domestic violence cases needs to be reexamined. More culturally congruent options should be made available.

This study's use of a community-based random sample resulted in the inclusion of a wider scope of women, including those who have not sought outside assistance. Because only a small fraction of battered women use shelters or the criminal justice system (or both), more studies should investigate the coping and psychological distress of battered women who have not sought assistance. One of the study's limitations was the use of measures that had not previously been validated. This is a common dilemma in research with previously understudied populations, for whom few, if any, existing scales have been validated. Socioculturally rooted manifestations of domestic violence necessitated the construction of a measure for the study population (Yoshihama, 1999). Measuring the effectiveness of specific coping strategies retrospectively, the relatively small number of items (6 active 
and 4 passive strategies), and binomial responses (ever used vs. never) are potential sources of mismeasurement. Drawing from the rich body of knowledge in the field of stress and coping, continuous efforts are needed to develop conceptually and psychometrically sound measures of coping that are relevant to populations with diverse cultural backgrounds and life experiences.

Despite the use of behavior-specific measures, underreporting of the experiences of domestic violence, and that of coping responses to a lesser degrees, is possible. It is also possible that the significant relationship between country of birth, the selection and appraisal of coping strategies, and psychological distress may have been confounded by variables other than those accounted for (e.g., abusiveness ratings, the respondent's age, or the partner's ethnicity). Clearly, more systematic investigation is needed to understand the role individuals' cultural backgrounds play in the selection and perceived effectiveness of specific coping strategies among battered women of other cultural groups. Future studies should investigate the choice and perceived effectiveness of coping strategies and their relationship to psychological wellbeing among individuals with a common cultural background but who differ by country of origin or other characteristics, e.g., Mexico-born versus U.S.-born individuals of Mexican descent. This study examined the use and effectiveness of coping strategies in response to a specific type of stressordomestic violence. Future studies should also investigate whether similar patterns exist in choice and perceived effectiveness of coping strategies with respect to other types of stressors. Attention to both within-group and between-group variations in coping responses and well-being will help expand our knowledge about coping and resiliency and help develop intervention aimed at promoting well-being of people in diverse population groups.

\section{REFERENCES}

Allen, P. G. (1986). Violence and American Indian women. In M. C. Burns (Ed.), The speaking profits us: Violence in the lives of women of color (pp. 5-7). Seattle, WA: The Center for the Prevention of Sexual and Domestic Violence.

Billings, A. G., \& Moos, R. H. (1981). The role of coping responses and social resources in attenuating the stress of life events. Journal of Behavioral Medicine, 2, 139-157.

Bowker, L. H., \& Maurer, L. (1986). The effectiveness of counseling services utilized by battered women. Women and Therapy, 5, 65-82.

Browne, A. (1993). Violence against women by male partners: Prevalence, outcomes, and policy implications. American Psychologist, 48, 1077-1087.

Derogatis, L. R. (1994). SCL-90-R: Administration, scoring, and procedures manual (3rd ed.). Minneapolis, MN: National Computer Systems. 
Derogatis, L. R., Richels, K., \& Rock, A. F. (1976). The SCL-90 and the MMPI: A step in the validation of a new self-report scale. British Journal of Psychiatry, 128, 280-289.

Finn, J. (1985). The stresses and coping behavior of battered women. Social Casework, 66, 341-349.

Folkman, S. (1984). Personal control and stress and coping processes: A theoretical analysis. Journal of Personality and Social Psychology, 46, 839-852.

Folkman, S., \& Lazarus, R. S. (1980). An analysis of coping in a middle-aged community sample. Journal of Health and Social Behavior, 21, 219-239.

Folkman, S., Schaefer, C., \& Lazarus, R. S. (1979). Cognitive processes as mediator of stress and coping. In V. Hamilton \& D. M. Warburton (Eds.), Human stress and cognition (pp. 265298). New York: Wiley.

Forsythe, C. J., \& Compas, B. E. (1987). Interaction of cognitive appraisals of stressful events and coping: Testing the goodness of fit hypothesis. Cognitive Therapy and Research, 11, $473-485$.

Ginorio, A., \& Reno, J. (1986). Violence in the lives of Latina women. In M. C. Burns (Ed.), The speaking profits us: Violence in the lives of women of color (pp. 13-16). Seattle, WA: Center for the Prevention of Sexual and Domestic Violence.

Hamby, S. L., \& Gray-Little, B. (1997). Responses to partner violence: Moving away from deficit model. Journal of Family Psychology, 11, 339-350.

Ho, C. K. (1990). An analysis of domestic violence in Asian American communities: A multicultural approach to counseling. Women and Therapy, 9, 129-150.

Holahan, C. J., \& Moos, R. H. (1990). Life stressors, resistance factors, and improved psychological functioning: An extension of the stress resistance paradigm. Journal of Personality and Social Psychology, 58, 909-917.

Holahan, C. J., \& Moos, R. H. (1991). Life stressors, personal and social resources, and depression: A 4-year structural model. Journal of Abnormal Psychology, 100, 31-38.

Horton, A. L., \& Johnson, B. L. (1993). Profile and strategies of women who have ended abuse. Families in Society, 74, 481-492.

Hudson, W., \& McIntosh, S. (1981). The assessment of spouse abuse: Two quantifiable dimensions. Journal of Marriage and the Family, 43, 873-885, 888.

Hyman, A., Schillinger, D., \& Lo, B. (1995). Laws mandating reporting of domestic violence: Do they promote patient well-being? Journal of the American Medical Association, 273, 1781-1787.

Kantor, G. K., Jasinski, J. L., \& Aldarondo, E. (1994). Sociocultural status and incidence of marital violence in Hispanic families. Violence and Victims, 9, 207-222.

Kelly, L. (1988). How women define their experiences of violence. In K. Yllo \& M. Bograd (Eds.), Feminist perspectives on wife abuse (pp. 114-132). Newbury Park, CA: Sage.

Kemp, A., Green, B., Hovanitz, C., \& Rawlings, E. (1995). Incidence and correlates of posttraumatic stress disorder in battered women: Shelter and community samples. Journal of Interpersonal Violence, 10, 43-55.

Kessler, R. C., McGonagle, K. A., Zhao, S., Nelson, C. B., Hughes, M., Eshleman, S., et al. (1994). Lifetime and 12-month prevalence of DSM-III-R psychiatric disorders in the United States: Results from the National Commodity Survey. Archives of General Psychiatry, 51, 8-19.

Lai, T. A. (1986). Asian women: Resisting the violence. In M. C. Burns (Ed.), The speaking profits us: Violence in the lives of women of color (pp. 8-11). Seattle, WA: Center for the Prevention of Sexual and Domestic Violence.

Marin, G., \& Marin, B. V. (1991). Research with Hispanic populations. Newbury Park, CA: Sage.

Marshall, L. L. (1992). Development of the severity of violence against women scales. Journal of Family Violence, 7, 103-121.

Mills, L. (1999). Killing her softly: Intimate abuse and the violence of state intervention. Harvard Law Review, 113, 551-613.

Mitchell, R. E., \& Hodson, C. A. (1983). Coping with domestic violence: Social support and psychological health among battered women. American Journal of Community Psychology, $11,629-654$. 
Moos, R. H., \& Schaefer, J. A. (1993). Coping resources and processes: Current concepts and measures. In L. Goldberger \& S. Breznitz (Eds.), Handbook of stress: Theoretical and clinical aspects (2nd ed., pp. 234-257). New York: Free Press.

Nurius, P. S., Furrey, J., \& Berliner, L. (1992). Coping capacity among women with abusive partners. Violence and Victims, 7, 229-243.

Pape, K. T., \& Arias, I. (1995). Control, coping, and victimization in dating relationships. Violence and Victims, 10, 43-54.

Pearlin, L. I., \& Schooler, C. (1978). The structure of coping. Journal of Health and Social Behavior, 19, 2-21.

Richie, B. E., \& Kanuha, V. (1993). Battered women of color in public health care systems: Racism, sexism and violence. In B. Blair \& S. E. Cayleff (Eds.), Wings of gauze: Women of color and the experience of health and illness (pp. 288-299). Detroit, MI: Wayne State University Press.

Robins, L. N., Locke, B., \& Regier, D. A. (1991). An overview of psychiatric disorders in America. In L. N. Robins \& D. A. Regier (Eds.), Psychiatric disorders in America: The Epidemiologic Catchment Area Study (pp. 328-386). New York: Free Press.

Russell, D. E., \& Howell, N. (1983). The prevalence of rape in the United States revised. Signs, $8,688-695$.

Schechter, S. (1996). The battered women's movement in the United States: New directions for institutional reform. In J. L. Edleson \& Z. C. Eisikovits (Eds.), Future interventions with battered women and their families (pp. 53-66). Thousand Oaks, CA: Sage.

Smith, M. D. (1987). The incidence and prevalence of woman abuse in Toronto. Violence and Victims, 2, 173-187.

Sorenson, S. B., \& Telles, C. A. (1991). Self-reports of spousal violence in a Mexican-American and non-Hispanic white population. Violence and Victims, 6, 3-15.

Straus, M. A. (1990). The Conflict Tactics Scale and its critics: An evaluation and new data on validity and reliability. In M. A. Straus \& R. J. Gelles (Eds.), Physical violence in American families: Risk factors and adaptations to violence in 8,145 families (pp. 49-73). New Brunswick, NJ: Transaction Publications.

Straus, M. A., \& Gelles, R. J. (1986). Societal change and change in family violence from 1975 to 1985 as revealed by two national surveys. Journal of Marriage and the Family, 48, 465-479.

Sullivan, C. M. (1991). Battered women as active helpseekers. Violence Update, 1, 1, 8, $10-11$.

Takeuchi, D. T., Kuo, H.-S., Kim, K., \& Leaf, P. J. (1990). Psychiatric symptom dimensions among Asian Americans and Native Hawaiians: An analysis of the Symptom Checklist. Journal of Community Psychology, 17, 319-329.

Tolman, R. M. (1989). The development of a measure of psychological maltreatment of women by their male partners. Violence and Victims, 4, 159-177.

Torres, S. (1987). Hispanic-American battered women: Why consider cultural differences? Response, 10, 20-21.

Uehara, E. S., Takeuchi, D. T., \& Smukler, M. (1994). Effects of combining disparate groups in the analysis of ethnic differences: Variations among Asian American mental health service consumers in level of community functioning. American Journal of Community Psychology, 22, 83-99.

Valentiner, D. P., Holahan, C. J., \& Moos, R. H. (1994). Social support, appraisals of event controllability, and coping: An integrative model. Journal of Personality and Social Psychology, 66, 1094-1102.

Wilson, M., \& Daly, M. (1993). Spousal homicide risk and estrangement. Violence and Victims, $8,3-16$.

Wyatt, G. E. (1985). The sexual abuse of Afro-American and white American women in childhood. Child Abuse and Neglect, 9, 507-519.

Yoshihama, M. (1999). Domestic violence against women of Japanese descent in Los Angeles: Two methods of estimating prevalence. Violence Against Women, 5, 869897. 
Yoshihama, M. (2000). Reinterpreting strength and safety in socio-cultural context: Dynamics of domestic violence and experiences of women of Japanese descent. Children and Youth Services Review, 22, 205-227.

Yoshihama, M. (2001). Model minority demystified: Emotional costs of multiple victimizations in the lives of women of Japanese descent. Journal of Human Behavior in the Social Environment, 3, 201-224.

Yoshihama, M., \& Sorenson, S. B. (1994). Physical, sexual, and emotional abuse by male intimates: Experiences of women in Japan. Violence and Victims, 9, 63-77.

Zorza, J., \& Woods, L. (1994). Analysis and policy implications of the new police domestic violence studies. New York: National Center on Women and Family Law. 